\title{
Neurochemical phenotype and function of endomorphin 2-immunopositive neurons in the myenteric plexus of the rat colon
}

\section{Jun-Ping $\mathrm{Li}^{1,2+}$, Xi-Yu Wang ${ }^{3}$, Chang-Jun Gao ${ }^{1+}$, Yong-Hui Liao ${ }^{1+}$, Juan $\mathrm{Qu}^{1}{ }^{1}$, Zhong-Yi He${ }^{2}$, Ting Zhang ${ }^{1}$, Guo-Du Wang ${ }^{3 *}$ and Yun-Qing Li $^{1 *}$}

${ }^{1}$ Department of Anatomy, Histology and Embryology, K.K. Leung Brain Research Centre, The Fourth Military Medical University, Xi'an, China

${ }^{2}$ Department of Anatomy, Histology and Embryology, Ningxia Medical University, Yinchuan, China

${ }^{3}$ Department of Physiology and Cell Biology, Medical Center, Ohio State University, Columbus, OH, USA

\section{Edited by:}

Javier DeFelipe, Cajal Institute,

Spain

Reviewed by:

L. Ashley Blackshaw, University of

Adelaide, Australia

Anthony Krantis, University of

Ottawa, Canada

${ }^{*}$ Correspondence:

Guo-Du Wang, Department of

Physiology and Cell Biology,

Medical Center, Ohio State

University, 303 Hamilton Hall, Neil

Avenue, Columbus, OH 43231, USA

e-mail:wang.772@osu.edu;

Yun-Qing Li, Department of

Anatomy, Histology and

Embryology, K.K. Leung Brain

Research Centre, The Fourth

Military Medical University, No.

169, West Changle Road, Xi'an,

710032 Shaanxi, China

e-mail: deptanat@fmmu.edu.cn

${ }^{\dagger}$ These authors have contributed

equally to this work.
The distribution and activity of endomorphins (EMs), which are endogenous $\mu$-opioid receptor (MOR) ligands in the gastrointestinal tract (GI), are yet to be elucidated. The current study aimed to shed light on this topic. EM2 was expressed in the enteric neurons in the myenteric plexus of the mid-colon. Of the EM2-immunoreactive (EM2-IR) neurons, $53 \pm 4.6 \%, 26 \pm 4.5 \%, 26 \pm 2.8 \%$ and $49 \pm 4.2 \%$ displayed immunopositive staining for choline acetyl transferase (ChAT), substance $P$ (SP), vasoactive intestinal peptide (VIP) and nitric oxide synthetase (NOS), respectively. A bath application of EM2 (2 $\mu \mathrm{M})$ enhanced spontaneous contractile amplitude and tension, which were reversed by $\beta$-FNA (an antagonist of MOR) but not NG-nitro-L-arginine methyl ether (L-NAME, a non-selective inhibitor of NOS) or VIP6-28 (an antagonist of the VIP receptor) in the colonic strips. EM2 significantly suppressed inhibitory junction potentials (IJPs) in 14 of the 17 examined circular muscle cells, and this effect was not antagonized by preincubation in L-NAME. EM2 was widely expressed in interneurons and motor neurons in the myenteric plexus and presynaptically inhibited fast IJPs, thereby enhancing spontaneous contraction and tension in the colonic smooth muscle.

Keywords: endomorphin-2, myenteric plexus, $\mu$-opioid receptor, inhibitory neuromuscular transmission, colonic motility

\section{INTRODUCTION}

Opioids play a significant role in mediating intestinal smooth muscle activity via the $\mu$-opioid receptor (MOR). Previous studies have shown that MOR is expressed in distinct neuronal populations, including Dogiel type I myenteric neurons such as motor neurons and interneurons responsible for gastrointestinal (GI) motility in the myenteric plexus (Bagnol et al., 1997; Ho et al., 2003; Sternini et al., 2004; DeHaven-Hudkins et al., 2008). The activation of MOR in the enteric nervous system (ENS) leads to decreased GI propulsive activity (Holzer, 2004; Webster et al., 2008). Orally administered morphine, a non-selective exogenous MOR agonist, typically inhibits GI propulsive activity, thereby resulting in constipation (Pappagallo, 2001; Camilleri, 2011; Kapoor, 2012; Labianca et al., 2012). A substantial body of evidence has demonstrated that colonic retention time is $80 \%$ of the oroanal transit time in animals, including humans, suggesting that morphine-induced constipation is likely of colonic origin (Banta et al., 1979; Arhan et al., 1981). However, the mechanism underlying this side effect of morphine has yet to be adequately elucidated, and no therapy to antagonize this side effect has been approved to date (Nishiwaki et al., 1998; Holzer, 2007, 2009; DeHaven-Hudkins et al., 2008; Leppert, 2010; Camilleri, 2011; Diego et al., 2011; Wein, 2012; Bader et al., 2013; Rauck, 2013).

Endomorphin-1 (Tyr-Pro-Trp-Phe-Nh2, EM1) and endomorphin-2 (Tyr-Pro-Phe-Phe-Nh2, EM2), the two endogenous ligands of MOR, were isolated from the bovine and human brain in 1997 (Zadina et al., 1997). EM1 and EM2 selectively bind to MOR at a high affinity (Zadina et al., 1997). EM1-immunoreactive (IR) and EM2-IR neuronal cell bodies are principally located in the hypothalamic nuclei, the nucleus tractus solitarii, the dorsal root ganglia (DRG) and the vagal ganglia in the CNS and the peripheral nervous system (PNS; Barr and Zadina, 1999; Martin-Schild et al., 1999; Pierce and Wessendorf, 2000; Niu et al., 2009). Both EM1 and EM2 are involved in the regulation of somatic and visceral information transmission. $\mu$-opioid receptor is the most abundantly expressed opioid receptor in the GI tract, especially the colon (Banta et al., 
1979; Nishimura et al., 1986). However, the distributions of EM1 and EM2 in the ENS and their effects on colonic motility are yet to be determined (Sternini et al., 2004). The cellular mechanisms by which MOR modulates intestinal motility also require further elucidation. Our pilot immunofluorescence histochemical staining experiments revealed that myenteric neurons in the rat colon express EM2 but not EM1. Organ bath measurements revealed that exogenous EM2 exerts two direct effects on colonic motility: EM2 enhances spontaneous contractile amplitude and tension and suppresses electrical field stimulation (EFS)-evoked twitch contraction in colonic preparations. Elucidating the mechanism by which EM2 stimulates colonic motility was the primary aim of the present study. We hypothesized that EM attenuates or facilitates colonic motility via the activation of opioid receptors in myenteric nerve cells. Therefore, we investigated the effects of endogenous opioids on colonic motility to enhance our understanding of EM-evoked bidirectional modulatory mechanisms and to provide novel insight into a potential therapeutic strategy in the treatment of GI dysfunction.

\section{MATERIALS AND METHODS ANIMALS}

Male Sprague-Dawley rats weighing 220-250 g were provided by the Experimental Animal Center of the Fourth Military Medical University (Xi'an, China). All protocols described below were approved by the Committee of Animal Use for Research and Education of the Fourth Military Medical University. All efforts were made to minimize the number of animals used and their suffering in accordance with the ethical guidelines for animal research (Zimmermann, 1983). After the rats were anesthetized using ether, the mid-colons between the right and left flexures were removed.

\section{IMMUNOFLUORESCENCE HISTOCHEMISTRY}

For whole-mount colonic preparations, the mucosa, submucosa and inner circular muscle layer from the tissues were separated from the outer longitudinal muscle layer, which was attached to the myenteric plexus.

Whole-mount colonic preparations from seven rats $(n=7)$ were processed for immunofluorescence histochemical doublestaining for EM2 and neuron-specific enolase (NSE), choline acetyl transferase (ChAT), substance $\mathrm{P}$ (SP), vasoactive intestinal peptide (VIP) or nitric oxide synthetase (NOS, the key enzyme for the synthesis of nitric oxide). The whole-mount sections were sequentially incubated in the following solutions: (1) a mixture of rabbit antiserum against EM2 (AB5104, 1:200; Chemicon, Billerica, MA, USA) and mouse antiserum against NSE (MAB314, 1:200; Chemicon), goat antiserum against ChAT (IHCR1008-6, 1:500; Chemicon), rat antiserum against SP (MAB356, 1:500; Chemicon), mouse antiserum against VIP (SC-25347, 1:200; Santa Cruz, Santa Cruz, CA, USA) or mouse antiserum against NOS (N2280, 1:2000; Sigma, Saint Louis, MO, USA) in 0.01 M phosphate-buffered saline (PBS, pH 7.4) containing 5\% (v/v) normal goat serum (NGS), 0.3\% (v/v) Triton X-100, 0.05\% (w/v) $\mathrm{NaN}_{3}$ and $0.25 \%(\mathrm{w} / \mathrm{v})$ carrageenan (PBS-NGS, $\mathrm{pH}$ 7.4) for $72 \mathrm{~h}$ at $4^{\circ} \mathrm{C}$; (2) a mixture of biotinylated donkey anti-rabbit
IgG (BA-1000, 1:200; Vector, Burlingame, CA, USA) for EM2 and Alexa 594-labeled donkey anti-mouse IgG (A21203, 1:500; Invitrogen, Grand Island, NY, USA) for NSE, VIP or NOS, Alexa 594-labeled donkey anti-goat IgG (A-11058, 1:500; Invitrogen) for ChAT- or Cy3-labeled donkey anti-rat IgG (AP189C, 1:200; Chemicon) for SP in PBS-NGS for $6 \mathrm{~h}$ at $4^{\circ} \mathrm{C}$; and (3) fluorescein isothiocyanate (FITC)-labeled avidin D (A-2001, $1: 1,000$; Vector) in PBS containing $0.3 \%$ Triton X-100 (PBS$\mathrm{X}, \mathrm{pH}$ 7.4) for $4 \mathrm{~h}$ at room temperature. Finally, the sections were cover-slipped using 0.01 M PBS containing 50\% (v/v) glycerin and $2.5 \%(\mathrm{w} / \mathrm{v})$ triethylene diamine (an anti-fading reagent).

\section{CONTROL EXPERIMENTS}

The rabbit antiserum against EM2 was prepared against the synthetic peptide Tyr-Pro-Phe-Phe-NH2 from full-length EM2 conjugated to BSA. We evaluated the specificity of this antibody by incubating it in either $2 \mu \mathrm{M}$ EM2 or $2 \mu \mathrm{M}$ EM1 peptide, as previously described (Huidobro-Toro and Way, 1981; Niu et al., 2009). Normal mouse sera were used to confirm the specificities of the mouse antibodies against NSE, VIP and NOS. Normal goat and rat sera were used to confirm the specificities of the goat antibody against ChAT and the rat antibody against SP, respectively, via replacement tests. In the present study, when the EM2 antibody was pre-absorbed using either homologous or heterologous synthetic peptides and when normal mouse, goat or rat sera were used to replace the mouse, goat or rat antibodies against NSE, VIP and NOS, ChAT and SP, respectively, no immunopositive staining was detected in our preparations. Therefore, the antibodies were considered to be specific and reliable (data not shown).

\section{CELL COUNTING}

After immunofluorescence histochemical staining, all of the fluorescence-labeled sections were observed under a confocal laser scanning microscope (Olympus FV1000; Tokyo, Japan) using the appropriate filters for FITC (excitation $490 \mathrm{~nm}$; emission $520 \mathrm{~nm}$ ) and Alexa 594 (excitation $590 \mathrm{~nm}$; emission $617 \mathrm{~nm}$ ). To estimate the extent of co-localization between EM2 and NSE, as well as VIP or NOS, three sets of representative whole-mount sections from the mid-colon were selected $(n=7)$. Plexuses were randomly selected for this analysis from each specimen. A ganglion that displayed a clear boundary and a neuronal cell body outline was randomly selected from each specimen for this analysis. In each specimen, approximately 6-7 ganglia were selected to count the number of various neuronal cell bodies; the total number of ganglia was approximately 35 in each set. Within each plexus, we determined the number of EM2-IR neuronal cell bodies, the number of neuronal cell bodies IR for other markers, and the number and percentage of neuronal cell bodies that expressed both markers.

\section{MECHANICAL EXPERIMENTS}

The isolated mid-colon $(1.0 \mathrm{~cm})$ was rapidly transferred to a dish for dissection. This colon segment was opened along the mesenteric border and pinned (mucosa side up) to a Sylgard base (Dow Corning, Midland, MI, USA). The mucosa layer was removed, and the preparations were sliced into muscle strips in the circular 
or longitudinal axis. These test strips were placed in $10 \mathrm{ml}$ of organ bath containing modified Krebs solution bubbled with 95\% $\mathrm{O}_{2}$ and $5 \% \mathrm{CO}_{2}$ at $37.0 \pm 0.5^{\circ} \mathrm{C}$, which was replaced every 20-30 min. An isometric force transducer (Harvard VF-1 Harvard Apparatus, Holliston, MA, USA) connected to a computer via an amplifier was used to record the motility of the muscle strips. These data were digitalized $(25 \mathrm{~Hz})$ using the Data 2001 software (Panlab, Barcelona, Spain) coupled to an A/D converter and were stored in the computer. The tension was set to $1 \mathrm{~g}$ to minimize local reflex stimulation of the bowel, and the strips were allowed to equilibrate for $1 \mathrm{~h}$. Electrical field stimulation $(0.5 \mathrm{~ms}, 10 \mathrm{~Hz}$, and $0.5 \mathrm{~mA}$ ) was applied using platinum electrodes connected via stimulus isolation units (Grass SIN5) to a square wave stimulator (Grass 588, Grass, Quincy, MA, USA). The concentration of all reagents is presented as the final concentration in $10 \mathrm{ml}$ of organ bath.

\section{INTRACELLULAR MICROELECTRODE RECORDINGS IN CIRCULAR MUSCLE}

The intracellular electrophysiological recording methods were previously described (Wang et al., 2007). In brief, "sharp" glass microelectrodes were used to record stimulus-evoked inhibitory junction potentials (IJPs) in the circular muscle coat of the mucosa-free preparations in which a portion of the circular muscle bands was removed to expose the myenteric plexus for the placement of the stimulation electrode. The preparations were pinned to Sylgard resin at the bottom of a $2.0-\mathrm{ml}$ recording chamber. The chamber was perfused at a rate of $10-15 \mathrm{ml} / \mathrm{min}$ with Krebs solution warmed to $37^{\circ} \mathrm{C}$ and gassed with $95 \% \mathrm{O}_{2}-5 \% \mathrm{CO}_{2}$ to buffer this solution at $\mathrm{pH}$ 7.3-7.4. During electrophysiological recording, $1 \mu \mathrm{M}$ nifedipine and scopolamine were added to the Krebs solution to suppress muscle movements. The microelectrodes, which displayed resistances of 80-120 M $\Omega$, were filled with $\mathrm{KCl}(2 \mathrm{M})$ or potassium acetate $(4 \mathrm{M})$. For the intraneuronal injection of electrical current, the preamplifier (M-767; World Precision Instruments, Sarasota, FL, USA) was equipped with a bridge circuit. The Grass SD9 stimulator (Grass Instrument Division, Astro-Med, Warwick, RI, USA) injected rectangular constant-current pulses. The electrometer output was amplified and observed using oscilloscopes (Tektronix 3012, Tektronix, Beaverton, OR, USA). Digital charts of the synaptic events and membrane potentials were recorded on the computer using the Data Acquisition System and LabChart software (ADInstruments, Colorado Springs, CO, USA). The bipolar insulated tungsten stimulating electrodes were connected via stimulus isolation units (Grass SIN5) to the Grass SD9 stimulator and were placed perpendicularly to the longitudinal axis of the preparation. In the myenteric plexus, the focal electrical stimulation of interganglionic fiber tracts induces neuromuscular junction potentials. The stimulus parameters were single pulses at an amplitude of $0.2 \mathrm{~mA}$ and a duration of $2 \mathrm{~ms}$.

\section{SOLUTIONS AND DRUGS}

The composition of Krebs solution was (in mM): $120 \mathrm{NaCl}$, $6 \mathrm{KCl}, 2.5 \mathrm{CaCl}_{2}, 1.2 \mathrm{MgCl}_{2}, 1.35 \mathrm{NaH}_{2} \mathrm{PO}_{4}, 14.4 \mathrm{NaHCO}_{3}$, and 11.5 glucose. The reagents used in this study were EM2 and $\beta$-FNA hydrochloride (Sigma), NG-nitro-L-arginine methyl ether, hydrochloride (L-NAME, Sigma), nifedipine, scopolamine (Sigma), tetrodotoxin (TTX, Taizhou Kante Biological Co., Ltd., Taizhou, Jiangsu, China), and the VIP fragment VIP $6-28$ (Sigma). The preparation of all stock solutions and their subsequent dilutions was performed using $0.9 \%$ saline. All concentrations are expressed as their final concentration in the organ bath and the perfusing chamber for intracellular recording.

\section{DATA ANALYSIS}

Statistical comparisons of the data sets were performed via ANOVA followed by Dunnett's post hoc test, and " $n$ " indicates the number of samples. Student's $t$-test was used to determine significance, and $P<0.05$ was considered to be significant.

\section{RESULTS IMMUNOFLUORESCENCE HISTOCHEMICAL STAINING}

The EM2-IR products were predominantly localized to the cytoplasm of the neuronal cell bodies in the ganglia and in the nerve fibers surrounding the ganglia. EM2 expression was detected at both of these sites in the myenteric plexus of the rat midcolon. EM2-IR markers were also detected outside the plexus. The EM2-IR nerve fibers formed a dense network between the neuronal cell bodies of the ganglia, and both varicose and nonvaricose fibers ran along the internodal strands between the bowel muscle and the myenteric plexus (Figure 1A). The EM2IR neurons typically displayed an oval cell body in which many thick dendrites protruded from the somata with a long axonal process that extended for a distance of $100 \mu \mathrm{M}$ or longer. A few EM2-IR neuronal cell bodies outside the primary meshes of the plexus were occasionally identified. The areas of these neuronal cell bodies were $35.5 \pm 10.8 \mu \mathrm{M}$ (length) by $17.8 \pm 4.6 \mu \mathrm{M}$ (width) (Figure 1B). Approximately $10.55 \pm 4.2$ EM2-IR neurons were detected in each ganglion.

Antisera against the general neuronal marker NSE (Mulderry et al., 1988) were used to label all neuronal components of the myenteric plexus, including the neuronal cell bodies and the varicose and non-varicose nerve fibers. Of the NSE-IR neurons, $57 \%$ were stained with EM2 in the myenteric ganglia (Figures 1C-E; Table 1). Next, we examined the co-localization between EM2 and ChAT, SP, VIP and NOS. ChAT-IR was predominantly localized to the somata and the nerve fibers, and $53 \pm 4.6 \%$ of the EM2-IR neuronal cell bodies were co-labeled with ChAT. EM2-IR fibers ran near the ChAT-IR neurons, and some of these fibers were even distributed around the ChATIR neuronal cell bodies (Figure 2A; Table 1). SP-IR was intense in the nerve fibers, which ran amid the EM2-IR neuronal cell bodies. Approximately $26 \pm 4.5 \%$ of the EM2-IR neurons were co-labeled with SP (Figure 2B; Table 1). VIP-IR neuronal cell bodies were occasionally detected in the myenteric plexus. The VIP-IR neurons displayed a slightly oval-shaped cell body and one long process as well as several short and lamellar processes surrounding the perikarya; $26 \pm 2.8 \%$ of the EM2-IR neuronal cell bodies were co-labeled with VIP (Figure 2C; Table 1). The NOS-IR products displayed a homogeneous distribution in the neuronal cell bodies without nuclear labeling, and $49 \pm 4.2 \%$ of the EM2-IR neurons were co-labeled with NOS (Figure 2D; Table 1). Based on immunofluorescence histochemical staining 

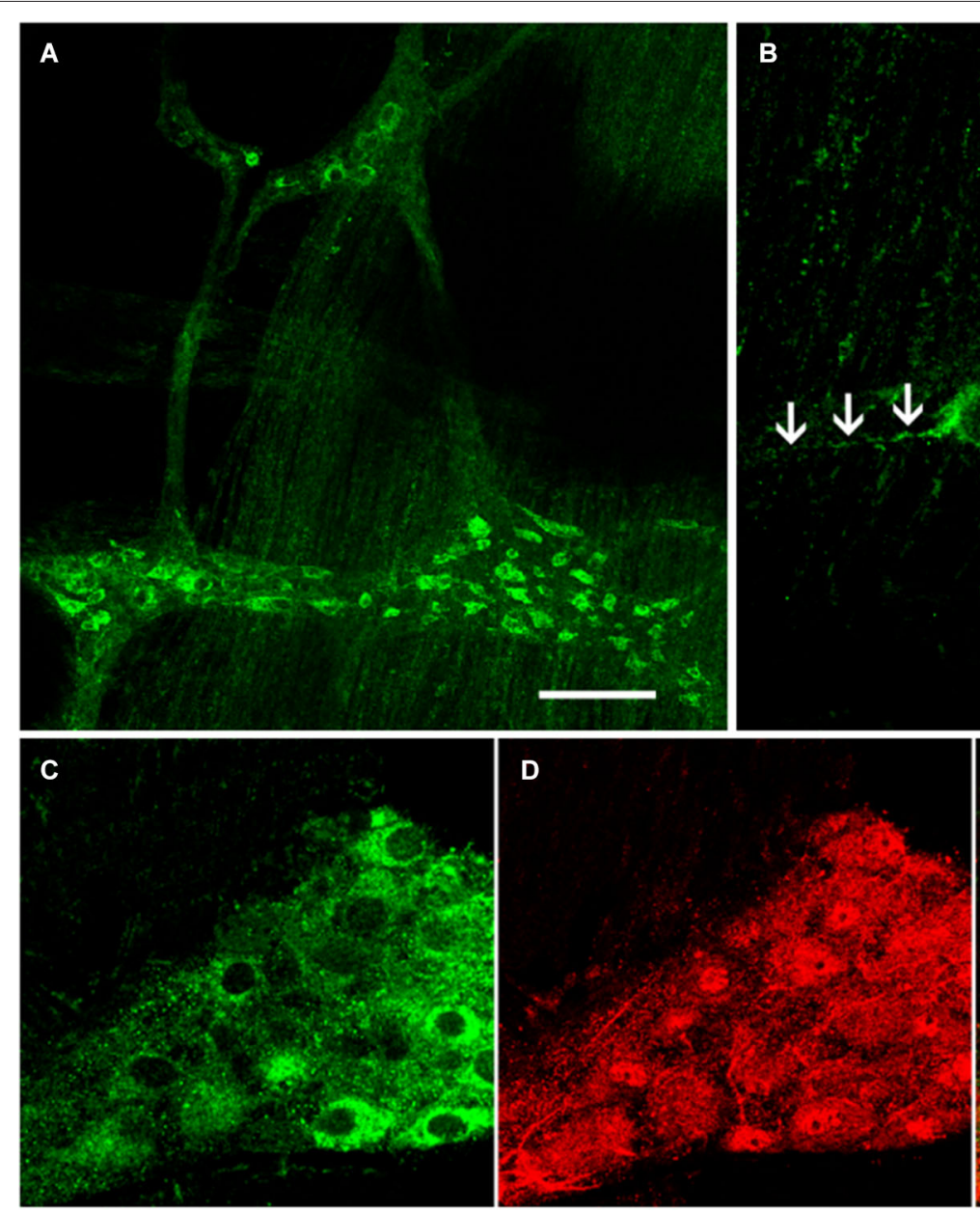
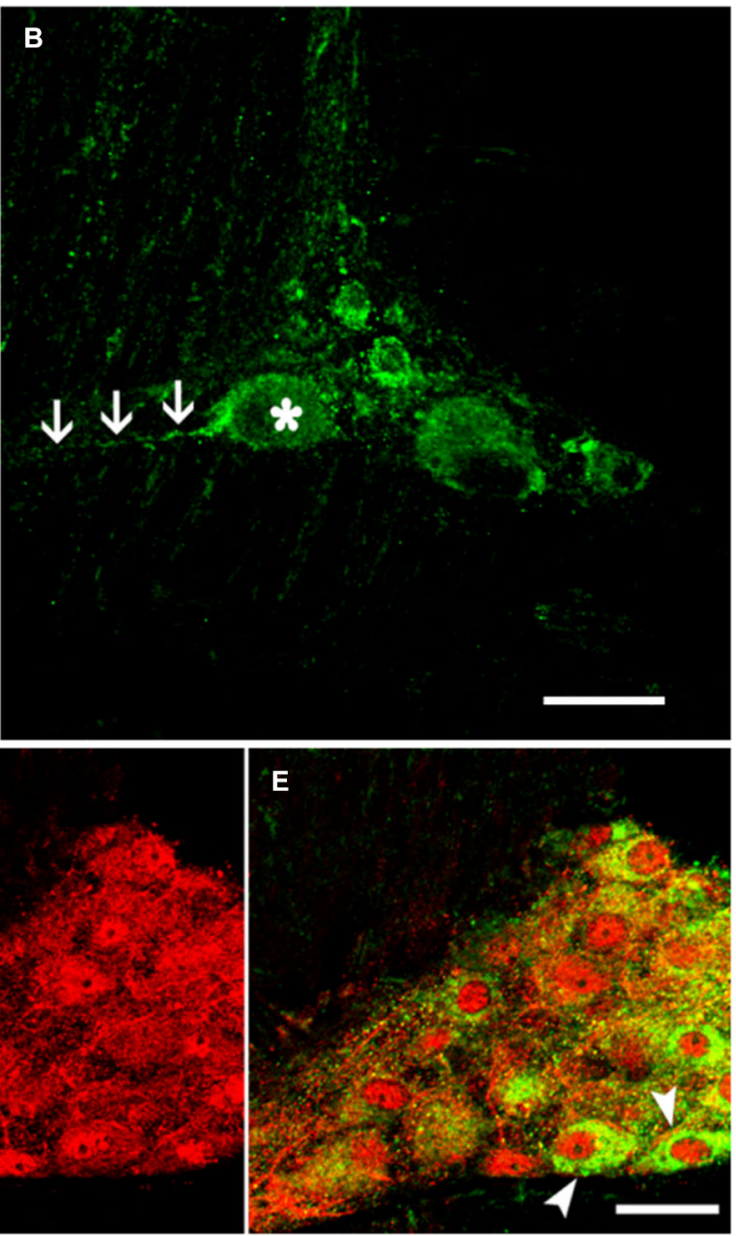

FIGURE 1 | Immunofluorescence histochemistry of whole-mount sections of the myenteric plexus from the rat colon. (A) EM2-IR fibers formed a network between the ganglia. (B) EM2-IR neurons typically extended a distinctive long process, as indicated by the arrows. Double-label immunofluorescence staining revealed the colocalization of EM2 (C) with NSE (D). The composite images show neurons co-labeled with EM2 and NSE (E). The arrows indicate typical neurons that expressed both EM2 and NSE. Scale bar: $\mathbf{A}=180 \mu \mathrm{M} ; \mathbf{B}, \mathbf{C}, \mathbf{D}$ and $\mathbf{E}=30 \mu \mathrm{M}$. (except for that in the EM2-IR neurons), only the NOS-IR processes at their origins could be detected, although these processes may not have extended far. Additionally, some EM2-IR nerve cell bodies were surrounded by VIP-IR and NOS-IR varicosities and terminals (Figures 2A,B). EM2 immunoreactivity was detected in $74 \pm 4.7 \%$ of the ChAT-IR neurons (Figure 2A; Table 1) and in $88 \pm 2.5 \%$ of the SP-IR neurons (Figure 2B; Table 1). Among the VIP-IR and NOS-IR neurons, nearly all displayed EM2 immunoreactivity (Figures 2C,D; Table 1).

\section{EFFECTS OF EM2 ON COLONIC MOTILITY}

The colonic muscle strips were mounted in an organ bath chamber to determine the effects of EM2 on intestinal motility. Regular cyclic spontaneous contractions occurred after incubation in Krebs solution for one half hour (24 preparations/6 rats). Bath application of EM2 (2 $\mu \mathrm{M})$ enhanced the amplitude of the spontaneous contractile waves in six circular and six longitudinal muscle strips (Figures 3, 4A,B).
The positive effects of EM2 on spontaneous contractions were not altered in the presence of $300 \mu \mathrm{M}$ L-NAME, a non-selective inhibitor of $\mathrm{NO}$ synthase, or $0.5 \mu \mathrm{M} \mathrm{VIP} 6-28$, a VIP receptor antagonist. The application of the selective MOR antagonist $\beta$-FNA $(5 \mu \mathrm{M})$ reversed the positive effects of EM2 on colonic contraction (Figures 3, 4A,B). Tetrodotoxin $(1 \mu \mathrm{M}), \mathrm{a} \mathrm{Na}^{+}$channel blocker, completely blocked the effects of EM2 on the muscle strips but partially suppressed spontaneous motility (results not shown).

Electrical field stimulation evoked twitch contractile waves in the colonic strips (Figures 3, 4Aa). The exposure of the strips to EM2 $(2 \mu \mathrm{M})$ partially suppressed the EFS-evoked contractile waves $(n=16$; Figures 3, 4Ab). The depressive effect of EM2 on the EFS-evoked contractile waves was abolished by $\beta$-FNA $(5 \mu \mathrm{M}$; Figures 3, 4Ae,C). Preincubation in either L-NAME $(300 \mu \mathrm{M})$ or $\mathrm{VIP}_{6-28}(0.5 \mu \mathrm{M})$ did not significantly alter the suppressive effects of EM2 on EFS-evoked twitch contraction (Figures 3, 4Ac,d,C). 

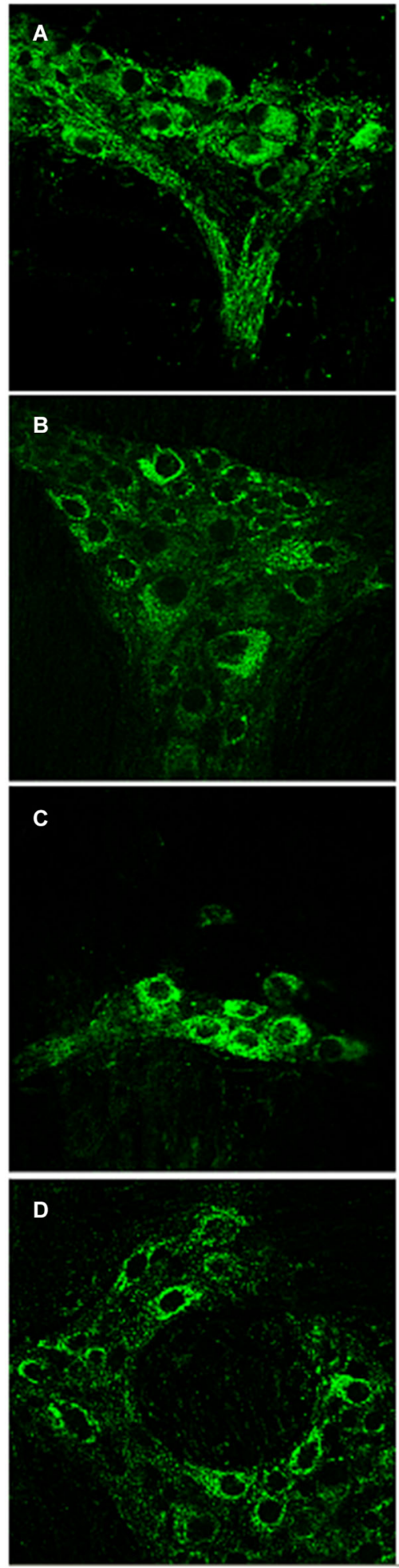

D'

FIGURE 2 Double-label immunofluorescence of whole-mount section showing the colocalization of EM2 (A, B, C, D) with ChAT $\left(A^{\prime}\right)$, SP ( $\left.B^{\prime}\right)$,

VIP ( $\left.\mathbf{C}^{\prime}\right)$ or NOS ( $\left.\mathbf{D}^{\prime}\right)$. The composite images show neurons co-labeled with

'B'

C'
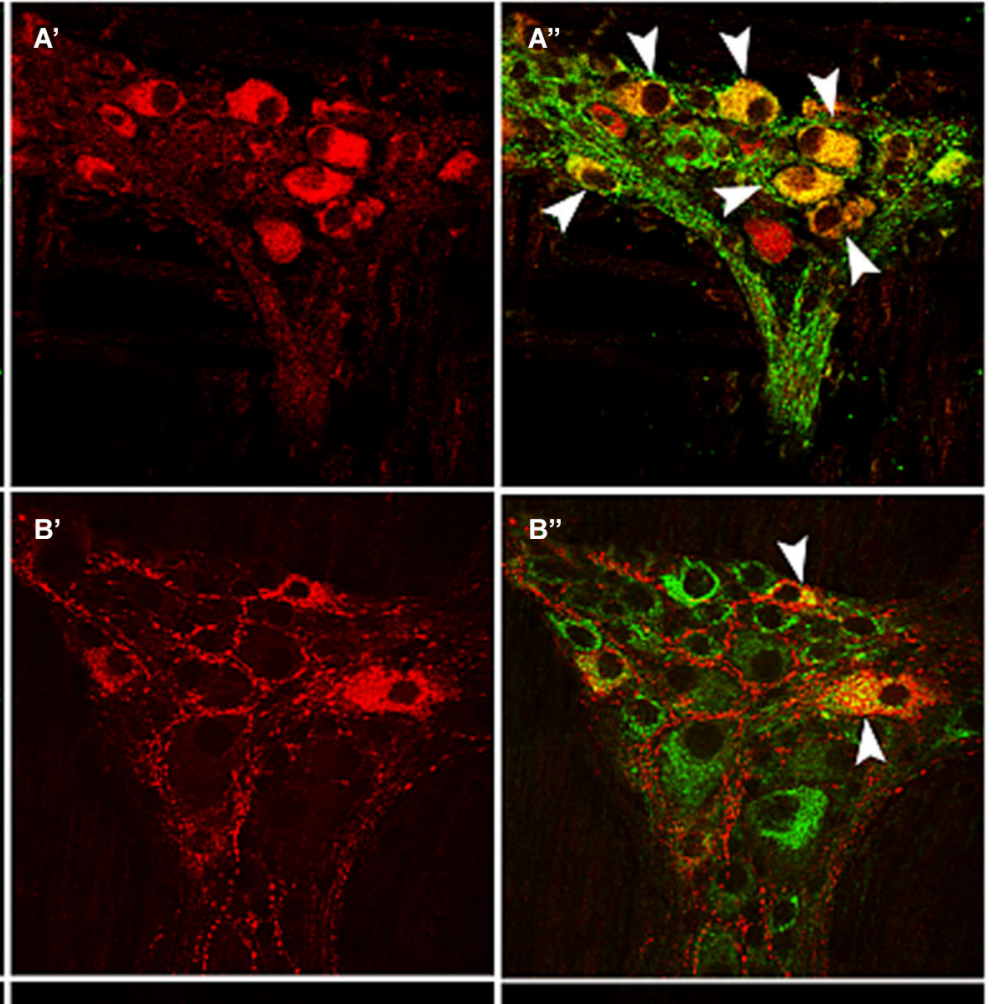

$C^{\prime \prime}$
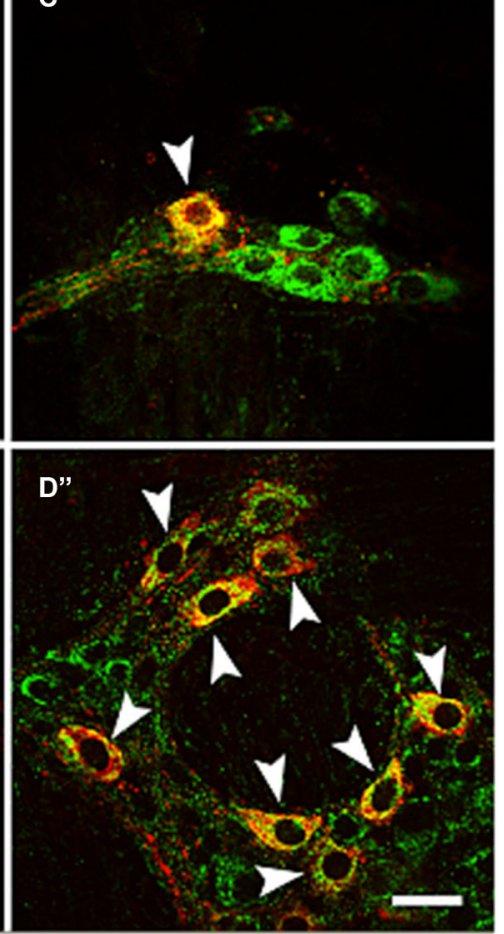

EM2 and ChAT ( $\left.\mathbf{A}^{\prime \prime}\right)$, SP (B"), VIP (C") or NOS (D"). The arrows indicate

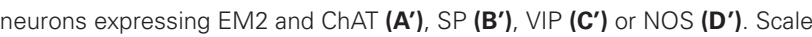
bar $=30 \mu \mathrm{M}$ 
Table 1 | Percentage of the co-localization of EM2 with various markers in myenteric neurons

\begin{tabular}{|c|c|c|c|}
\hline Marker pairs & $\begin{array}{l}\text { Numbers of myenteric ganglion } \\
\text { neurons (mean } \pm \text { SD) }\end{array}$ & $\begin{array}{l}\text { Numbers of doubly labeled } \\
\text { ganglion neurons (mean } \pm \text { SD) }\end{array}$ & Percentages $(\%)($ mean \pm S.E.M) \\
\hline ChAT (n1)/EM2 (n2) & $7.23 \pm 3.6 / 9.85 \pm 3.8$ & $\begin{array}{l}\text { ChAT of EM2 (n1/n2) } \\
\text { EM2 of ChAT (n2/n1) }\end{array}$ & $\begin{array}{l}53 \pm 4.6 \\
74 \pm 4.7\end{array}$ \\
\hline NOS (n3)/EM2 (n2) & $6.67 \pm 2.5 / 13.89 \pm 4.8$ & $\begin{array}{l}\text { NOS of EM2 (n3/n2) } \\
\text { EM2of NOS (n2/n3) }\end{array}$ & $\begin{array}{c}49 \pm 4.2 \\
100.0\end{array}$ \\
\hline NSE (n4)/EM2 (n2) & $20.45 \pm 5.9 / 11.45 \pm 3.6$ & $\begin{array}{l}\text { NSE of EM2 }(n 4 / n 2) \\
\text { EM2 of NSE (n2/n4) }\end{array}$ & $\begin{array}{c}100.0 \\
57 \pm 4.1\end{array}$ \\
\hline VIP (n6)/EM2 (n2) & $2.5 \pm 0.8 / 10.4 \pm 3.8$ & $\begin{array}{l}\text { VIP of EM2 }(n 6 / n 2) \\
\text { EM2 of VIP }(n 2 / n 6)\end{array}$ & $\begin{array}{c}26 \pm 2.8 \\
100.0\end{array}$ \\
\hline
\end{tabular}

\section{INTRACELLULAR RECORDINGS IN CIRCULAR MUSCLE CELLS}

To explore the cellular mechanisms by which EM2 modulates colonic motility, intracellular recordings were performed to examine the synaptic responses of neuromuscular junctions and the potential changes in the circular muscle of seven colonic myenteric preparations. Both spontaneous excitatory junction potentials (EJPs) and IJPs were detected in the colonic circular muscle cells in the presence of Krebs solution lacking nifedipine (an L-type calcium channel blocker) and scopolamine (a competitive antagonist of M-type muscarinic receptors). Perfusion with EM2 $(2 \mu \mathrm{M})$ suppressed either EJPs or IJPs in 22/26 circular muscle cells from nine colonic myenteric preparations. Nevertheless, the recordings did not remain stable in the muscle cells for an extended period (commonly $<10 \mathrm{~min}$ ) because strong muscle movement resulted in the dislocation of the microelectrode from the recorded muscle cells. Under these recording conditions, EFS also evoked rapid and strong movements that terminated the recordings. Because previous evidence
A

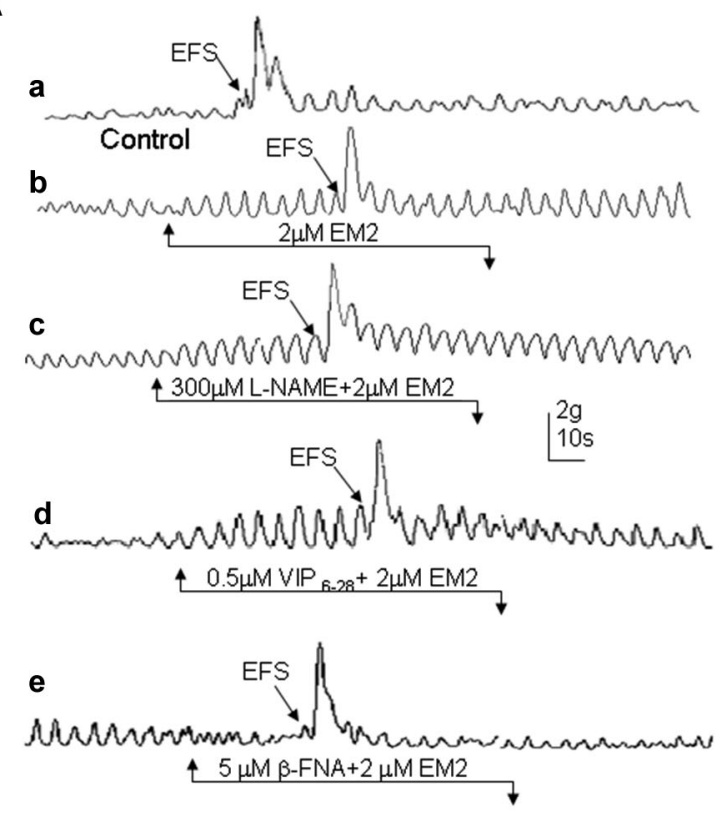

B

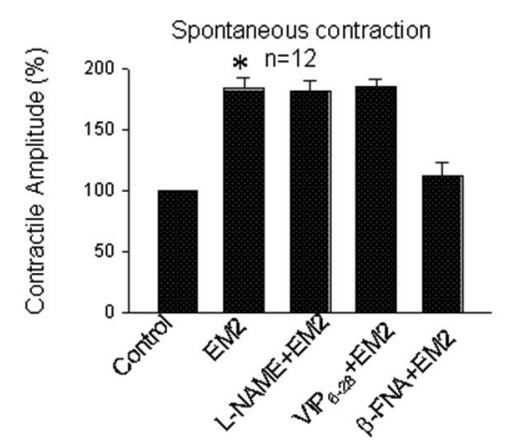

C

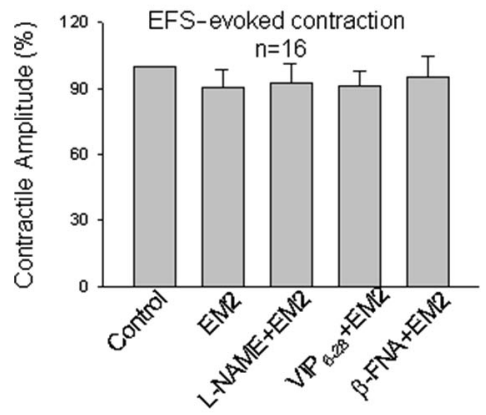

FIGURE 3 | Effects of EM2 on the motility of circular muscle strips from the colon. (A) Spontaneous contractile amplitude and EFS-evoked contractions (a). A bath application of EM2 enhanced the spontaneous contractile amplitude and slightly suppressed EFS-evoked contractions (b). The presence of both L-NAME (an antagonist of NO) and $\mathrm{VIP}_{6-28}$ (a VIP receptor antagonist) did not significantly alter the effects of EM2 (c,d). $\beta$-FNA (an antagonist of MOR) blocked the EM2-induced changes in the strips (e)

(B) Summary of the effects of EM2 on spontaneous contractions.

(C) Summary of EFS-evoked contractions. ${ }^{*} P<0.05$. 
A

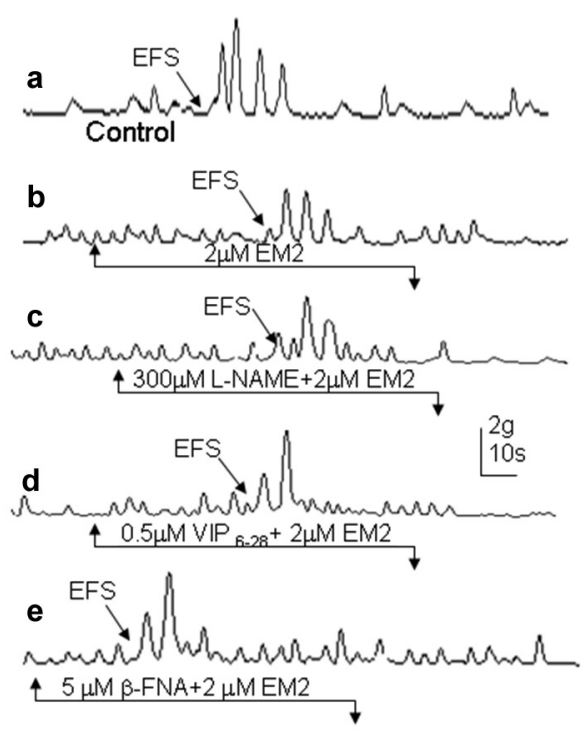

B

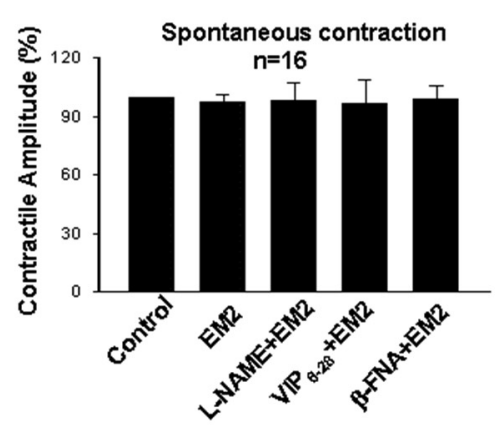

C

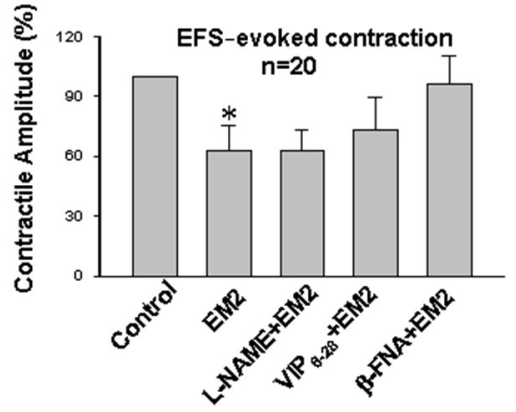

FIGURE 4 | Effects of EM2 on the motility of longitudinal muscle strips from the colon. (A) Spontaneous contractile amplitude and electrical field stimulation (EFS)-evoked contractions (a). Exposure of the strips to EM2 inhibited EFS-evoked contraction but did not appear to affect the spontaneous contractile amplitude (b). The presence of both L-NAME and VIP $\mathrm{P}_{6-28}$ did not significantly alter the effects of EM2 (c,d). $\beta$-FNA blocked the EM2-induced changes in the strips (e). (B) Summary of the effects of EM2 on spontaneous contractions. (C) Summary of EFS-evoked contractions. ${ }^{*} P<0.05$. has demonstrated that opioids inhibit intestinal movement via presynaptic MORs to reduce acetylcholine release (Fichna et al., 2007), the present investigation focused on the effects of EM2 on inhibitory neuromuscular transmission in these muscle cells. When Krebs solution containing nifedipine $(1 \mu \mathrm{M})$ and scopolamine $(1 \mu \mathrm{M})$ was employed to inhibit muscle movement, EFSevoked IJPs were stably recorded from the circular muscle cells. The application of EM2 $(2 \mu \mathrm{M})$ significantly suppressed the amplitude of the EFS-evoked IJPs at an $\mathrm{IC}_{50}$ of $0.35 \pm 0.13 \mu \mathrm{M}$ $(n=16$; Figures $5 \mathrm{~A}, \mathbf{B})$. The pre-application of $\beta$-FNA $(10 \mu \mathrm{M})$ markedly ameliorated the inhibitory effect of EM2 on these IJPs (Figures $5 \mathrm{~A}, \mathbf{B})$. However, the application of $\beta$-FNA $(10 \mu \mathrm{M})$ alone did not exert any clear effect on the IJPs. To examine the effects of EM2 on the nitric oxide- and VIP-mediated slow phase of the IJPs, the NOS inhibitor L-NAME and the VIP receptor antagonist $\mathrm{VIP}_{6-28}$, respectively, were used. Neither LNAME $(300 \mu \mathrm{M})$ nor $\operatorname{VIP}_{6-28}(0.2 \mu \mathrm{M})$ exerted a significant effect on the EM2-mediated inhibition of the IJPs (Figures 5A,B).

\section{DISCUSSION}

Neurons in the myenteric plexus are located between the circular and longitudinal muscle layers. Myenteric neurons extend projection fibers to the circular and longitudinal muscle layers (Grider and Foxx-Orenstein, 1999), forming a widely distributed neuronal network that regulates intestinal muscle tension and propulsion (Sarna, 2006). Gastrointestinal tract motility disorders induced by opioids are likely of colonic origin and mediated via MOR, such that EM, a potent endogenous MOR agonist, may participate in the modulation of GI motility. However, the mechanisms by which EM modulates GI motility are yet to be identified despite recent morphological and functional findings (Fichna et al., 2007). In the present study, we tested the hypothesis that EM inhibits colonic motility via the activation of opioid receptors in myenteric neurons by combining immunostaining, organ bath and intracellular recording methods to obtain morphological, physiological and pharmacological data. It is helpful to understand the mechanism by which EM2 modulates colonic motility. The major findings in the present study include the expression of EM2 in myenteric neurons and the EM2-mediated suppression of colonic motility via the activation of presynaptic MORs to inhibit inhibitory and excitatory neuromuscular transmission.

\section{DISTRIBUTION AND CHARACTERISTICS OF EM2-IR NEURONS IN THE MYENTERIC PLEXUS OF THE RAT COLON}

In the present study, immunofluorescence histochemical staining revealed that most (57\%) EM2-IR neuronal cell bodies and fibers formed a dense network that was restricted to the colonic myenteric plexus. These EM2-IR neurons are also immunopositive for ChAT, SP, VIP or NOS.

Previous studies showed that ChAT-expressing neurons correspond to ascending excitatory motor neurons that innervate the muscle and that some populations of ascending and descending interneurons also express ChAT (Costa et al., 1996). Substance $\mathrm{P}$ acts on smooth muscle directly or indirectly by modulating the release of excitatory transmitters from nerve fibers (Scheurer et al., 1994). VIP-IR is localized to both ascending and descending interneurons, as well as to descending 
A
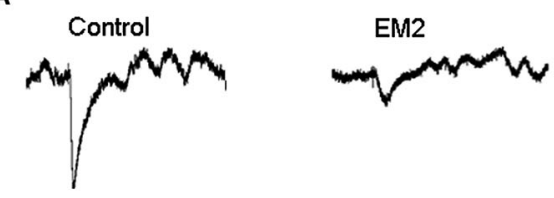

L-NAME + EM2
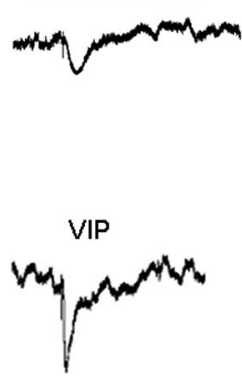
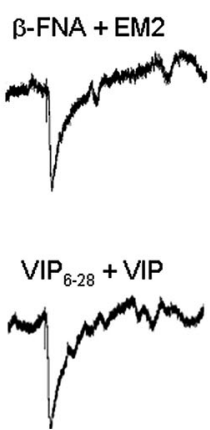

FIGURE 5 | EM2 inhibits inhibitory neuromuscular transmission in the circular muscle of the colon. (A) A bath application of EM2 significantly suppressed IJPs. The effect of EM2 on the IJPs was antagonized via pretreatment with $\beta$-FNA but not L-NAME. The

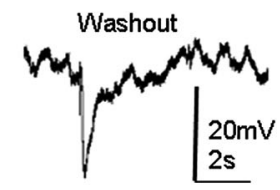

B

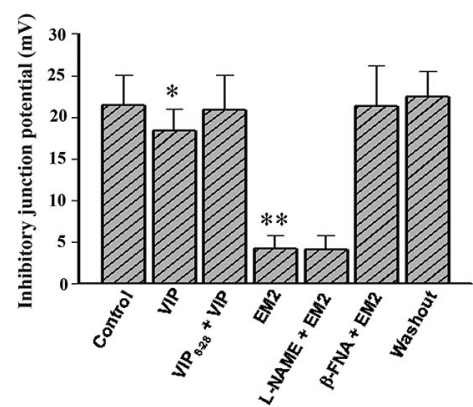

application of VIP slightly reduced the IJPs, which was reversed via pretreatment with $V I P_{6-28}$. (B) Summary of the inhibitory effect of EM2 on electrical stimulation-evoked IJPs in the circular muscle of the colon. ${ }^{*} P<0.05$ and ${ }^{*} P<0.01$. inhibitory motor neurons (Costa et al., 2000; Lomax and Furness, 2000; Smith et al., 2007). Additionally, NOS has been detected in both circular and longitudinal inhibitory motor neurons and in descending interneurons (Schemann and Neunlist, 2004). ChAT-IR, VIP-IR and NOS-IR neurons in the myenteric plexus (Fickel et al., 1997; Benabdallah et al., 2008) but not the smooth muscle of the rat intestine (Fickel et al., 1997; Porcher et al., 1999; Fichna et al., 2007) expressed MORs (Fickel et al., 1997; Ho et al., 2003). At the cellular level, MORs are predominantly localized to the cell surface of motor neurons and interneurons and to the nerve fibers that innervate the longitudinal and circular muscles (Ho et al., 2003). Our morphological findings indicated that EM2 colocalized with both VIP and NO in the perikarya of myenteric neurons, suggesting that EM2-IR neurons correspond to a subpopulation of myenteric interneurons and/or motor neurons.

\section{EFFECTS OF EM2 ON COLONIC MOTILITY}

In the current study, we demonstrated that EM2 partially inhibited EFS-evoked contraction waves and that this was reversed by the MOR antagonist $\beta$-FNA. Enteric excitatory motor neurons are commonly thought to release excitatory transmitters, such as cholinergic and tachykininergic neurotransmitters (Nedialkova et al., 2011). The finding that EM2 suppresses EFS-evoked colonic twitch contraction also suggests that EM2 plays an inhibitory role in the release of excitatory transmitters from these motor neurons.

However, EM2 enhanced the spontaneous contractile amplitude, which was also reversed by the MOR antagonist $\beta$-FNA. This finding suggests that EM2 directly activates MORs on motor neurons to suppress neurotransmitter release into the colonic strips. We also found that TTX, a neuronal $\mathrm{Na}^{+}$channel blocker, completely blocked the effects of EM2 on the muscle strips but did not abolish its effects on spontaneous contraction, indicating that the effects of EM2 on colonic motility involve neurogenic modulation. The spontaneous contractions of colonic segments are induced and maintained by an increase in the tonus (Benabdallah et al., 2008), which is regulated by noncholinergic non-adrenergic neurotransmitters, including $\mathrm{NO}$ and VIP (Benabdallah et al., 2008). Tonically released NO from myenteric neurons inhibits increases in spontaneous motility both in vitro and in vivo (Gil et al., 2010), and VIP inhibits the spontaneous mechanical activity induced by the cyclic depolarization of the circular muscle (Plujà et al., 2000). The NO-induced inhibitory tone contributes to rhythmic contractions, which produce the mixing and propulsive movements in the fasting and the postprandial states (Sarna, 2006). L-NAME and $\mathrm{VIP}_{6-28}$ exerted no clear effect on the EM2-induced enhancement of spontaneous muscle strip contractions. These results suggest that the effect of EM2 on colonic motility is not due to the neuronal or muscular activities of NO or VIP. Accumulating evidence has demonstrated that non-cholinergic non-adrenergic inhibitory neuromuscular transmission contains purinergic, nitrergic and VIP-ergic components and that the purinergic component plays the primary role in inhibiting transmission (Sarna, 2006; Wang et al., 2007).

$\mu$-opioid receptors are known to modulate pre-and postsynaptic neurotransmission, thereby inhibiting neural activity in the ENS. The present study is the first to report that EM2 activates MORs to enhance colonic motility in rats; this is consistent with other findings from mice (Yu et al., 2007; Wang et al., 2008). The dual effects of EM2 to attenuate and facilitate bowel contractions suggest that EM2 performs a bidirectional modulation of colonic motility. 


\section{INTRACELLULAR MICROELECTRODE RECORDINGS IN CIRCULAR MUSCLE}

The electrophysiological results of the present study demonstrated that exogenous EM2 significantly suppressed fast IJPs but did not significantly affect slow IJPs. The pre-application of the selective MOR antagonist $\beta$-FNA reversed the inhibitory effects of EM2 on the IJPs. Immunohistochemical evidence indicates that MORs are expressed in myenteric neurons (Fickel et al., 1997; Ho et al., 2003) but not in intestinal smooth muscle (Fickel et al., 1997; Porcher et al., 1999). Previous morphological evidence is consistent with our results that EM2 exerts no direct effect on smooth muscle in the bowel but plays a neurogenic regulatory role in intestinal motility. The activation of presynaptic MORs has been reported to inhibit the release of acetylcholine and ATP in the intestinal submucosal plexus (Yun et al., 2004). We found that EM2 not only inhibits EFS-evoked fast and strong contractions but also enhances spontaneous contractile waves and tension in the bowel strips. Elucidating this facilitative effect of EM2 and its underlying mechanism were the primary aims of the present study. To explore these cellular mechanisms, neuropharmacology and electrophysiological recording were performed to examine neuromuscular transmission in the rat mid-colon. Pre-incubation in the MOR antagonist $\beta$-FNA specifically reversed this inhibitory effect of EM2. The present electrophysiological and neuropharmacological results demonstrated that the facilitative effect of EM2 on colonic motility occurs via a presynaptic mechanism, i.e., the suppression of the release of inhibitory transmitters, to augment smooth muscle contraction and tension. Previous evidence has shown that IJPs are generally biphasic and consist of an initial large-amplitude and rapidly activating component (fast IJP) followed by a smaller and more slowly activating component (slow IJP). The slow IJP is abolished by treatments that block the synthesis of nitric oxide. However, purinergic neurotransmitters (via activating $\mathrm{P}_{2} \mathrm{Y}_{1}$ receptors) are responsible for the primary fast component of non-cholinergic non-adrenergic IJPs (Wang et al., 2007; Gallego et al., 2012; Hwang et al., 2012; Goyal et al., 2013).

Taken together, the present results indicate the following: (1) EM2 acts as an endogenous opioidergic neurotransmitter in the rat colon; (2) EM2 activates MORs that are localized to ENS neurons and nerve terminals in the smooth muscle coat to modulate colonic motility; and (3) EM2 inhibits EFS-evoked muscle contractions, potentially by suppressing the release of prejunction excitatory neurotransmitters, and suppresses inhibitory ligand release to enhance spontaneous colonic contractions and tension. These inhibitory ligands do not include NO or VIP but may include purinergic inhibitory transmitters.

In conclusion, EM2-IR was found to be widely localized to the cell bodies of enteric neurons that also display immunoreactivity for ChAT-, NOS-, SP- or VIP in the myenteric plexus of the rat colon. EM2, an endogenous opioid, inhibits intestinal motility in accordance with our hypothesis and enhances colonic smooth muscle movement by decreasing or reducing inhibitory transmitter release into the bowel neuromuscular junction. This EM2-mediated bidirectional modulation of colonic motility may provide new insight into the mechanisms underlying GI protection and dysfunction, which may help us to establish a novel understanding of the opioidergic system in the GI tract and new therapeutic strategies for GI diseases.

\section{AUTHORS' CONTRIBUTIONS}

Morphological experiments: Jun-Ping Li, Chang-Jun Gao, YongHui Liao, Juan Qu and Zhong-Yi He. Electrophysiological experiments: Jun-Ping Li, Xi-Yu Wang and Guo-Du Wang. Technical support: Ting Zhang. Manuscript writing: Jun-Ping Li, Guo-Du Wang and Yun-Qing Li. Study conception and design: Yun-Qing Li.

\section{ACKNOWLEDGMENTS}

This work was supported by grants from the National Natural Science Foundation of China (Nos. 31010103909 and 81371239).

\section{REFERENCES}

Arhan, P., Devroede, G., Jehannin, B., Lanza, M., Faverdin, C., Dornic, C., et al. (1981). Segmental colonic transit time. Dis. Colon Rectum 24, 625-629. doi: 10. 1007/bf02605761

Bader, S., Dürk, T., and Becker, G. (2013). Methylnaltrexone for the treatment of opioid-induced constipation. Expert Rev. Gastroenterol. Hepatol. 7, 13-26. doi: 10.1586/egh.12.63

Bagnol, D., Mansour, A., Akil, H., and Watson, S. J. (1997). Cellular localization and distribution of the cloned mu and kappa opioid receptors in rat gastrointestinal tract. Neuroscience 81, 579-591. doi: 10.1016/s0306-4522(97)00227-3

Banta, C. A., Clemens, E. T., Krinsky, M. M., and Sheffy, B. E. (1979). Sites of organic acid production and patterns of digesta movement in the gastrointestinal tract of dogs. J. Nutr. 109, 1592-1600.

Barr, G. A., and Zadina, J. E. (1999). Maturation of endomorphin-2 in the dorsal horn of the medulla and spinal cord of the rat. Neuroreport 10, 3857-3860. doi: 10.1097/00001756-199912160-00025

Benabdallah, H., Messaoudi, D., and Gharzouli, K. (2008). The spontaneous mechanical activity of the circular smooth muscle of the rabbit colon in vitro. Pharmacol. Res. 57, 132-141. doi: 10.1016/j.phrs.2008.01.002

Camilleri, M. (2011). Opioid-induced constipation: challenges and therapeutic opportunities. Am. J. Gastroenterol. 106, 835-842. doi: 10.1038/ajg.2011.30

Costa, M., Brookes, S. J., and Hennig, G. W. (2000). Anatomy and physiology of the enteric nervous system. Gut 47(Suppl. 4), iv15-iv19. doi: 10.1136/gut.47. suppl_4.iv15

Costa, M., Brookes, S. J., Steele, P. A., Gibbins, I., Burcher, E., and Kandiah, C. J. (1996). Neurochemical classification of myenteric neurons in the guinea-pig ileum. Neuroscience 75, 949-967. doi: 10.1016/0306-4522(96)00275-8

DeHaven-Hudkins, D. L., DeHaven, R. N., Little, P. J., and Techner, L. M. (2008). The involvement of the mu-opioid receptor in gastrointestinal pathophysiology: therapeutic opportunities forantagonism at this receptor. Pharmacol. Ther. 117, 162-187. doi: 10.1016/j.pharmthera.2007.09.007

Diego, L., Atayee, R., Helmons, P., Hsiao, G., and von Gunten, C. F. (2011). Novel opioid antagonists for opioid-induced bowel dysfunction. Expert Opin. Investig. Drugs 20, 1047-1056. doi: 10.1517/13543784.2011.592830

Fichna, J., Janecka, A., Costentin, J., and Do Rego, J. C. (2007). The endomorphin system and its evolving neurophysiological role. Pharmacol. Rev. 59, 88-123. doi: $10.1124 /$ pr.59.1.3

Fickel, J., Bagnol, D., Watson, S. J., and Akil, H. (1997). Opioid receptor expression in the rat gastrointestinal tract: a quantitative study with comparison to the brain. Brain Res. Mol. Brain Res. 46, 1-8. doi: 10.1016/s0169-328x(96)00266-5

Gallego, D., Gil, V., Martínez-Cutillas, M., Mañé, N., Martín, M. T., and Jiménez, M. (2012). Purinergic neuromuscular transmission is absent in the colon of P2Y1 knocked out mice. J. Physiol. 590, 1943-1956. doi: 10.1113/jphysiol.2011.224345

Gil, V., Gallego, D., Grasa, L., Martín, M. T., and Jiménez, M. (2010). Purinergic and nitrergic neuromuscular transmission mediates spontaneous neuronal activity in the rat colon. Am. J. Physiol. Gastrointest. Liver Physiol. 299, G158-G169. doi: 10.1152/ajpgi.00448.2009

Goyal, R. K., Sullivan, M. P., and Chaudhury, A. (2013). Progress in understanding of inhibitory purinergic neuromuscular transmission in the gut. Neurogastroenterol. Motil. 25, 203-207. doi: 10.1111/nmo.12090 
Grider, J. R., and Foxx-Orenstein, A. E. (1999). Mediators and regulation of peristalsis. Curr. Opin. Gastroenterol. 15, 22-25. doi: 10.1097/00001574-19990100000005

Ho, A., Lievore, A., Patierno, S., Kohlmeier, S. E., Tonini, M., and Sternini, C. (2003). Neurochemically distinct classes of myenteric neurons express the muopioid receptor in the guinea pig ileum. J. Comp. Neurol. 458, 404-411. doi: 10. 1002/cne.10606

Holzer, P. (2004). Opioids and opioid receptors in the enteric nervous system: from a problem in opioid analgesia to a possible new prokinetic therapy in humans. Neurosci. Lett. 361, 192-195. doi: 10.1016/j.neulet.2003.12.004

Holzer, P. (2007). Treatment of opioid-induced gut dysfunction. Expert Opin. Investig. Drugs 16, 181-194. doi: 10.1517/13543784.16.2.181

Holzer, P. (2009). Opioid receptors in the gastrointestinal tract. Regul. Pept. 155, 11-17. doi: 10.1016/j.regpep.2009.03.012

Huidobro-Toro, J. P., and Way, E. L. (1981). Contractile effect of morphine and related opioid alkaloids, beta-endorphin and methionine enkephalin on the isolated colon from long evans rats. Br. J. Pharmacol. 74, 681-694. doi: 10.1111/j. 1476-5381.1981.tb10479.x

Hwang, S. J., Blair, P. J., Durnin, L., Mutafova-Yambolieva, V., Sanders, K. M., and Ward, S. M. (2012). P2Y1 purinoreceptors are fundamental to inhibitory motor control of murine colonic excitability and transit. J. Physiol. 590, 1957-1972. doi: 10.1113/jphysiol.2011.224634

Kapoor, S. (2012). Opioid/naloxone prolonged release combinations for opioid induced constipation. World J. Gastroenterol. 18, 3921-3922. doi: 10.3748/wig. v18.i29.3921

Labianca, R., Sarzi-Puttini, P., Zuccaro, S. M., Cherubino, P., Vellucci, R., and Fornasari, D. (2012). Adverse effects associated with non-opioid and opioid treatment in patients with chronic pain. Clin. Drug Investig. 32(Suppl. 1), 5363. doi: 10.2165/11630080-000000000-00000

Leppert, W. (2010). The role of opioid receptor antagonists in the treatment of opioid-Induced constipation: a review. Adv. Ther. 27, 714-730. doi: 10 1007/s12325-010-0063-0

Lomax, A. E., and Furness, J. B. (2000). Neurochemical classification of enteric neurons in the guinea-pig distal colon. Cell Tissue Res. 302, 59-72. doi: 10. 1007/s004410000260

Martin-Schild, S., Gerall, A. A., Kastin, A. J., and Zadina, J. E. (1999). Differential distribution of endomorphin1- and endomorphin 2-like immunoreactivities in the CNS of the rodent. J. Comp. Neurol. 405, 450-471. doi: 10.1002/(sici)10969861(19990322)405:4<450::aid-cne2>3.3.co;2-r

Mulderry, P. K., Ghatei, M. A., Spokes, R. A., Jones, P. M., Pierson, A. M., Hamid, Q. A., et al. (1988). Differential expression of alpha-CGRP and beta-CGRP by primary sensory neurons and enteric autonomic neurons of the rat. Neuroscience 25, 195-205. doi: 10.1016/0306-4522(88)90018-8

Nedialkova, N., Stavreva, G., Negrev, N., Ivancheva, C., and Radomirov, R. (2011) Functional coordination of motor activity in colonic smooth muscles in rat experimental model. Physiol. Res. 60, 659-666.

Nishimura, E., Buchan, A. M., and McIntosh, C. H. (1986). Autoradiographic localization of mu- and delta-type opioid receptors in the gastrointestinal tract of the rat and guinea pig. Gastroenterology 91, 1084-1094.

Nishiwaki, H., Saitoh, N., Nishio, H., Takeuchi, T., and Hata, F. (1998). Inhibitory effect of endomorphin-1 and -2 on acetylcholine release from myenteric plexus of guinea pig ileum. Jpn. J. Pharmacol. 78, 83-86. doi: 10.1254/jjp.78.83

Niu, L., Chen, T., Wang, Y. Y., and Li, Y. Q. (2009). Neurochemical phenotypes of endomorphin -2-containing neurons in vagal nodose neurons of the adult rat. Neurochem. Int. 55, 542-551. doi: 10.1016/j.neuint.2009.05.010

Pappagallo, M. (2001). Incidence, prevalence and management of opioid bowel dysfunction. Am. J. Surg. 182, 11S-18S. doi: 10.1016/s0002-9610(01) 00782-6

Pierce, T. L., and Wessendorf, M. W. (2000). Immunocytochemical mapping of endomorphin -2- immunoreactivity in rat brain. J. Chem. Neuroanat. 18, 181207. doi: 10.1016/s0891-0618(00)00042-9

Plujà, L., Fernández, E., and Jiménez, M. (2000). Electrical and mechanical effects of vasoactive intestinal peptide and pituitary adenylate cyclase-activating peptide in the rat colon involve different mechanisms. Eur. J. Pharmacol. 389, 217-224. doi: 10.1016/s0014-2999(99)00773-6
Porcher, C., Bagnol, D., and Watson, S. J. (1999). Opioid peptide mRNA expression in the colon of the rat. Neurosci. Lett. 272, 111-114. doi: 10.1016/s03043940(99)00549-2

Rauck, R. L. (2013). Treatment of opioid-induced constipation: focus on the peripheral $\mu$-opioid receptor antagonist methylnaltrexone. Drugs 73, 12971306. doi: 10.1007/s40265-013-0084-5

Sarna, S. K. (2006). Molecular, functional and pharmacological targets for the development of gut promotility drugs. Am. J. Physiol. Gastrointest. Liver Physiol. 291, G545-G555. doi: 10.1152/ajpgi.00122.2006

Schemann, M., and Neunlist, M. (2004). The human enteric nervous system. Neurogastroenterol. Motil. 16(Suppl. 1), 55-59. doi: 10.1111/j.1743-3150.2004 00476.x

Scheurer, U., Drack, E., and Halter, F. (1994). Substance P activates rat colonic motility via excitatory and inhibitory neural pathways and direct action on muscles. J. Pharmacol. Exp. Ther. 271, 7-13.

Smith, T. K., Spencer, N. J., Hennig, G. W., and Dickson, E. J. (2007). Recent advances in enteric neurobiology: mechanosensitive interneurons. Neurogastroenterol. Motil. 19, 869-878. doi: 10.1111/j.1365-2982.2007.01019.x

Sternini, C., Patierno, S., Selmer, I. S., and Kirchgessner, A. (2004). The opioid system in the gastrointestinal tract. Neurogastroenterol. Motil. 16(Suppl. 2), 3 16. doi: 10.1111/j.1743-3150.2004.00553.x

Wang, G. D., Wang, X. Y., Hu, H. Z., Liu, S., Gao, N., Fang, X., et al. (2007). Inhibitory neuromuscular transmission mediated by the $\mathrm{P} 2 \mathrm{Y} 1$ purinergic receptor in guinea pig small intestine. Am. J. Physiol. Gastrointest. Liver Physiol. 292, G1483-G1489. doi: 10.1152/ajpgi.00450.2006

Wang, C. L., Wang, X., Yu, Y., Cui, Y., Liu, H. M., Lai, L. H., et al. (2008). Type 1 diabetes attenuates the modulatory effects of endomorphins on mouse colonic motility. Neuropeptides 42, 69-77. doi: 10.1016/j.npep.2007.10.001

Webster, L., Jansen, J. P., Peppin, J., Lasko, B., Irving, G., Morlion, B., et al. (2008) Alvimopan, a peripherally acting mu-opioid receptor (PAM-OR) antagonist for the treatment of opioid-induced bowel dysfunction: results from a randomized, double-blind, placebo-controlled, dose-finding study in subjects taking opioids for chronic non-cancer pain. Pain 137, 428-440. doi: 10.1016/j.pain.2007.11.008

Wein, S. (2012). Opioid-induced constipation. J. Pain Palliat. Care Pharmacother. 26, 382-384. doi: 10.3109/15360288.2012.734907

Yu, Y., Cui, Y., Wang, X., Lai, L. H., Wang, C. L., Fan, Y. Z., et al. (2007). In vitro characterization of the effects of endomorphin 1 and 2, endogenous ligands for mu-opioid receptors, on mouse colonic motility. Biochem. Pharmacol. 73, 13841393. doi: 10.1016/j.bcp.2007.01.011

Yun, X., Wand, X. Y., Wang, G. D., Liu, S. M., Hu, H. Z., Gao, N., et al. (2004). Presynaptic mu-opioid receptors inhibit release of acetycholine and ATP in guinea-pig small intestinal submucosal plexus. Gastroenterology 126, A-11.

Zadina, J. E., Hackler, L., Ge, L. J., and Kastin, A. J. (1997). A potent and selective endogenous agonist for the mu-opiate receptor. Nature 386, 499-502. doi: 10 . 1038/386499a0

Zimmermann, M. (1983). Ethical guidelines for investigations of experimental pain in conscious animals. Pain 16, 109-110. doi: 10.1016/0304-3959(83)90201-4

Conflict of Interest Statement: The authors declare that the research was conducted in the absence of any commercial or financial relationships that could be construed as a potential conflict of interest.

Received: 13 June 2014; accepted: 22 November 2014; published online: 16 December 2014.

Citation: Li J-P, Wang X-Y, Gao C-J, Liao Y-H, Qu J, He Z-Y, Zhang T, Wang G$D$ and $L i$ Y-Q (2014) Neurochemical phenotype and function of endomorphin 2immunopositive neurons in the myenteric plexus of the rat colon. Front. Neuroanat. 8:149. doi: 10.3389/fnana.2014.00149

This article was submitted to the journal Frontiers in Neuroanatomy.

Copyright (C) 2014 Li, Wang, Gao, Liao, Qu, He, Zhang, Wang and Li. This is an openaccess article distributed under the terms of the Creative Commons Attribution License (CC BY). The use, distribution and reproduction in other forums is permitted, provided the original author(s) or licensor are credited and that the original publication in this journal is cited, in accordance with accepted academic practice. No use, distribution or reproduction is permitted which does not comply with these terms. 